\title{
Polyphosphoinositides Are Derived from Ether-linked Inositol Glycerophospholipids in Rat Brain
}

\author{
Sun H. Shin, Jong S. Kim, Hak R. Kim, Jin K. Lim, Byung K. Choi and Young K. Yeo* \\ Lipid Chemistry Laboratory, Kyungpook National University, Daegu, 702-701 Korea
}

Received 29 July 2004, Accepted 7 April 2005

\begin{abstract}
Membrane inositol glycerophospholipid (IGP) is metabolized to phosphatidylinositol-4-phosphate (PIP), phosphatidylinositol4, 5-bisphosphate $\left(\mathrm{PIP}_{2}\right)$, and inositol triphosphate $\left(\mathrm{IP}_{3}\right)$ in signaling transduction. This study was carried out to determine the subclasses of IGP involved in signaling pathway. The acyl chain moieties of the phospholipids are easily modulated by dietary fatty acids. We analyzed acyl chain composition of IGP 3-subclasses, PIP and PIP ${ }_{2}$ from rat brain after feeding sunflower seed oil enriched with linoleic acid or fish oil high in eicosapentaenoic acid and docosahexaenoic acid. Long chain polyunsaturated fatty acids (LCPUFA) as eicosapentaenoic acid and docosahexaenoic acid were not incorporated into ether-linked IGP (alkenylacylglycerophosphoinositol and alkylacyl-glycerophosphoinositol), PIP and PIP , while diacyl-glycerophosphoinositol (GPI) contained high LCPUFA. These results suggest that PIP might be phosphorylated from only the ether-linked IGP (alkenylacyl- and alkylacyl species) but not from diacyl subclass for signals to intracellular responses in the plasma membrane of rat brain.
\end{abstract}

Keywords: Docosapentaenoic acid, Docosahexaenoic acid, Ether-linked inositol glycerophospholipids, Phosphatidylinositol-4 phosphate, Phosphatidylinositol-4,5-bisphosphate, Polyphosphoinositides

\section{Introduction}

The functions and biochemical mechanisms involved in cell signalling by inositol glycerophospholipid (IGP) breakdown are now well defined (Low and Saltiel, 1988; Yasutomi, 1992; Berridge, 1993). Hydrolysis of phosphatidylinositol yields inositol triphosphate, which promotes the release of $\mathrm{Ca}^{2+}$ from intracellular stores and diacylglycerol, which activates protein kinase $\mathrm{C}$. The increase in cytosolic $\mathrm{Ca}^{2+}$ plays a major role in

*To whom correspondence should be addressed.

Tel: 82-53-950-5757; Fax: 82-53-957-0722

E-mail: ykyeo@knu.ac.kr the physiological responses observed with agonists that promote IGP hydrolysis, although the specific role of the protein kinase $\mathrm{C}$ activation still remains unclear. The phosphatidylinositol found to be most important in signal transduction were two phosphorylated derivatives of IGP, phosphatidylinositol-4-phosphate (PIP) and phosphatidylinositol4, 5-bisphosphate $\left(\mathrm{PIP}_{2}\right)$, which are thought to be located mainly in the inner leaflet of the plasma membrane. Although $\mathrm{PIP}_{2}$ is less plentiful in animal cell membranes than IGP, it is the hydrolysis of $\mathrm{PIP}_{2}$ that matters most.

The previous paper has established that the long chain polyunsaturated fatty acids (LCPUFA) profiles of PIP and $\mathrm{PIP}_{2}$ were little affected by dietary fats, whereas IGP was highly influenced by dietary fats (Alsted and Hoy, 1992). Thus it is important to study the fatty acid profiles of 3subclasses of IGP affected by dietary lipids to compare the profiles of PIP and $\mathrm{PIP}_{2}$. In most biological preparations there are usually 3-subclasses of IGP including two types of ether lipids. These ether lipids are alkenyl and alkyl analogs of IGP. The alkenyl moieties have a double bond adjacent to the ether linkage, whereas the alkyl moieties are saturated at this position. The alkenyl, alkyl and acyl chains may have different numbers of carbon atoms and double bonds (Curstedt, 1983).

Although the specific functions of the ether types of IGP have not been well-defined, they might have been associated with IGP pathway in signaling transduction. Thus, it is important to determine the role of ether-linked IGP in IGP hydrolysis during cell signaling.

\section{Materials and Methods}

Animal and diets Fourteen weanling female Sprague-Dawley rats (Korea Chemical Research Institute, Taejeon, Korea) weighing average $88.2 \mathrm{~g}$ were divided into two groups. One group was fed the diet containing $10 \%$ sunflower seed oil enriched in linoleic acid (18:2n-6) and the other was fed the diet containing 9\% fish oil containing high eicosapentaenoic acid and docosahexaenoic acid (Table 1), supplemented with sunflower seed oil to satisfy the 
Table 1. Composition of the sunflower seed oil (SO) and fish oil (FO) diets

\begin{tabular}{ccc}
\hline \multirow{2}{*}{ Ingredients } & \multicolumn{2}{c}{ Composition (wt \%) } \\
\cline { 2 - 3 } & SO & FO \\
\hline Casein $^{\mathrm{a}}$ & 20.0 & 20.0 \\
Choline chloride $^{\mathrm{b}}$ & 0.2 & 0.2 \\
Corn starch & 10.0 & 10.0 \\
Sucrose & 50.0 & 50.0 \\
vitamin mix $^{\mathrm{c}}$ & 1.0 & 1.0 \\
Mineral mix $^{\mathrm{d}}$ & 4.5 & 4.5 \\
Straw $_{\text {Sunflower seed oil }}^{\mathrm{e}}$ & 4.0 & 4.0 \\
Fish oil $^{\mathrm{f}}$ & 10.0 & 1.0 \\
\hline
\end{tabular}

aNamyang Milk Co., Kongju, Korea.

${ }^{\text {b} W a k o ~ P u r e ~ C h e m i c a l ~ I n d u s t r i e s ~ L t d ., ~ O s a k a, ~ J a p a n . ~}$

'Purchased from Taesung Institue, Euwang, Korea. Provided (mg/ $100 \mathrm{~g}$ diet): retinol, 6,012 IU; thiamin, 3; riboflavin, 5; pyridoxine, 1; cyanocobalamin, 2: ascorbicacid, 2: cholecalciferol, 2,000 IU: tocopherol, 3 IU: phytonadione, 1: folic acid, 0.1; biotin, 0.012; Ca pentothenate, 5: Nicotin amide, 5: DL-Methionine, 10: L-Lysine, 2: choline bitartrate, 20:

'Purchased from Taehan Pharmaceutical Co., Seoul, Korea. Provided (per kg): I, 150 mg; Mn, 200 mg; S, 4 g; Co, 100 mg; Fe, 2 g; Zn, 100 mg; Ni, 50 mg; Cu, 100 mg; Mg, 3 g Ca, 2 g; Se, $40 \mu \mathrm{g}$.

${ }^{\mathrm{e}}$ Extracted from sunflower seed.

fPurshased from Ewha Oil and Fat. Co. Ltd., Pusan, Korea.

nutritional requirements for $18: 2 \mathrm{n}-6$, for 4 weeks and allowed ad libitum access to water and diets in a room with controlled temperature $24-25^{\circ} \mathrm{C}$ and with $12 \mathrm{~h}$ dark-light cycle. The fatty acid compositions of the diets are shown in Table 2 . The rats were killed by decapitation. The brain from each rat were immediately excised, rinsed, perfused with $0.9 \%$ saline solution and weighed. Total lipids were extracted according to the method of Rahmant et al. (2002).

Analyses of lipids The phospholipid classes were seperated by thin layer chromatography on precoated silica gel 60 plates (E. Marck, Darmstadt, F.R.G.), using chloroform/methanol/acetic acid/ water $(50: 37.5: 3.5: 2, \mathrm{v} / \mathrm{v} / \mathrm{v} / \mathrm{v})$ as a solvent system (Holub and Skeaff, 1987) and visualized under ultraviolet light after spraying with 2',7'-dichloro-fluorescein solution (Sigma Chemical Co., St. Louis, USA).

The isolated IGP was converted to acetate derivatives following phospholypase C hydrolysis by the method of Bernett et al. (1985) in order to provide for the isolation of the alkenylacyl, alkylacyl, and diacyl species of the IGP. The resulting diacylglycerols were acetylated with $1 \mathrm{ml}$ of acetic anhydride and five drops of pyridine at $80^{\circ} \mathrm{C}$ for $1 \mathrm{~h}$ and subsequently separated by TLC using a solvent system of petroleum ether/diethyl ether/acetic acid $(90: 10: 1, \mathrm{v} / \mathrm{v} / \mathrm{v})$ followed by a second development in toluene (Yeo et al., 1989). The three subclasses of the IGP were visualized under ultraviolet light after expousure to $2^{\prime}, 7^{\prime}$-dichloro-fluorescein solution (Sigma Chemical Co., St. Louis, USA).

In order to seperate phosphatidylinositol-4-phosphate (PIP) and
Table 2. Fatty acid composition of the sunflower seed oil (SO) and fish oil (FO) diets

\begin{tabular}{ccc}
\hline & \multicolumn{2}{c}{ Composition $(\mathrm{mol} \%)$} \\
\cline { 2 - 3 } Fatty acid $^{\mathrm{a}}$ & $\mathrm{SO}$ & $\mathrm{FO}$ \\
\hline $14: 0$ & 0.4 & 4.3 \\
$16: 0$ & 10.5 & 14.9 \\
$16: 1$ & 0.1 & 8.5 \\
$18: 0$ & 5.7 & 4.5 \\
$18: 1(\text { iso })^{\mathrm{b}}$ & 27.6 & 22.8 \\
$18: 2 \mathrm{n}-6$ & 54.5 & 9.3 \\
$18: 3 \mathrm{n}-3$ & $\operatorname{tr}$ & 0.8 \\
$18: 4 \mathrm{n}-3$ & $\operatorname{tr}$ & 2.1 \\
$20: 1$ & $\operatorname{tr}$ & 9.5 \\
$20: 3 \mathrm{n}-6$ & 0.9 & $\operatorname{tr}$ \\
$20: 4 \mathrm{n}-6$ & $\operatorname{tr}$ & 0.2 \\
$20: 5 \mathrm{n}-3$ & $\operatorname{tr}$ & 11.7 \\
$22: 1$ & $\operatorname{tr}$ & 0.9 \\
$22: 6 \mathrm{n}-3$ & $\operatorname{tr}$ & 9.8 \\
\hline
\end{tabular}

${ }^{\mathrm{a}}$ Other minor fatty acids have been excluded from the table.

biso means isomers.

phosphatidylinositol-4, 5-bisphosphate ( $\left.\mathrm{PIP}_{2}\right)$, the TLC plates were soaked for $2 \mathrm{~min}$ in a $0.054 \mathrm{M}$ oxalic acid solution adjusted to $\mathrm{pH}$ 7.2 with $\mathrm{KOH}$, allowed to dry at room temperature and activated by heating at $110^{\circ} \mathrm{C}$ for $15 \mathrm{~min}$ (Chaffoy de Courcelles et al., 1984). The IGP from brain tissues and standard phospholipids (Sigma Chemical Co., St. Louis, USA) were spotted on different lanes 1.5 $\mathrm{cm}$ from the bottom of the divided plate. The first acidic solvent system was composed of chloroform/methanol/acetic acid $/ \mathrm{H}_{2} \mathrm{O}$ $(55: 43: 3: 4, \mathrm{v} / \mathrm{v} / \mathrm{v} / \mathrm{v})$. The development was stopped when the solvent front reached about $0.5 \mathrm{~cm}$ from the top of the plate. The second basic solvent system was composed of chroloform/ methanol/ammomium hydroxide/ $\mathrm{H}_{2} \mathrm{O}(40: 70: 10: 20, \mathrm{v} / \mathrm{v} / \mathrm{v} / \mathrm{v})$. The development was stopped when the solvent front reached $25 \%$ the distance of the first solvent front (Medh and Weigel, 1989). The fractions were visualized under ultraviolet light after spraying with 2',7'-dichlorofluorescein solution.

The IGP, 3-subclasses of IGP, PIP and $\mathrm{PIP}_{2}$ fractions were scraped from the plates and transmethylated with $6 \%$ methanolic sulfuric acid at $90^{\circ} \mathrm{C}$ for $1 \mathrm{~h}$ in the presence of known amounts of monopentadecanoin (Nu Chek Prep, Elysian, USA) as an internal standard to give subsequent mass determinations of the fatty acyl chains. The fatty acid compositions of the three subclasses from the IGP were analyzed by a Hewlett-Packard Model 5890 A gas chromatograph equipped with a DB-225 megabore column (Chromatographic Specialties, Brockville, Canada) and identified by comparison of their retention times with those of known standards (Nu Chek Prep, Elysian, USA). The flow rate of the nitrogen was $36 \mathrm{ml} / \mathrm{min}$ and the oven temperature during these isothermal runs was $210^{\circ} \mathrm{C}$. Since the acyl group composition of the two ether lipid subclasses are derived only from the sn- 2 position, while the acyl groups from the diacyl subclasses represent the sn-1 plus sn-2 positions, the molar amounts of total methyl esters derived from the two ether lipid subclasses were multiplied by 2 to compare them quantitatively with the diacyl subclasses. The results 
Table 3. Distribution of 3-subclasses of inositolglycerophospholipid in brain of animals fed either the sunflower seed oil (SO) or fish oil (FO) diets ${ }^{\mathrm{a}}$

\begin{tabular}{ccr}
\hline \multirow{2}{*}{$\begin{array}{c}\text { Phospholipid } \\
\text { subclass }\end{array}$} & \multicolumn{2}{c}{ Composition (mol \%) } \\
\cline { 2 - 3 } & \multicolumn{1}{c}{ SO } & \multicolumn{1}{c}{ FO } \\
\hline Alkenylacyl-GPI & $22.5 \pm 3.2$ & $20.6 \pm 4.1$ \\
Alkylacyl-GPI & $10.8 \pm 0.8$ & $8.7 \pm 1.2$ \\
Diacyl-GPI & $66.7 \pm 4.5$ & $70.7 \pm 4.5$ \\
\hline
\end{tabular}

${ }^{a}$ Values represent means \pm S.E. for seven animals/group.

were analyzed by using one-way analysis of variance for comparison of the sunflower seed oil or fish oil groups and expressed as $\mathrm{mol} \%$.

\section{Results}

The relative abundance of the three subclasses of the brain IGP are given in Table 3. The distributions in the sunflower seed oil animals were not significantly different from the fish oil animals. However, the relative distributions of the alkylacyl, alkenylacyl and diacyl subclasses from the IGP were considerably different among the 3-subclasses of IGP from the two dietary group (Table 3).

The compositions of the fatty acyl moieties in the IGP are provided in Table 4. The major unsaturated fatty acid in the IGP from the sunflower seed oil animals was arachidonic acid, with the fish oil animals being relatively more enriched than the sunflower seed oil animals (21.1 versus $26.7 \mathrm{~mol} \%$ ). The fatty acid composition of the IGP was considerably unique compared to CGP and EGP which were very dependent upon dietary fatty acids. Higher levels of arachidonic acid in the fish oil group might be remarkable only in IGP, although there was no enough arachidonic acid or its precursor contained in the fish oil diet. Docosahexaenoic acid level in the fish oil group doubled relative to that in the sunflower seed oil animals.

No docosahexaenoic acid was found in alkenylacyl- and alkylacyl-glycerophosphoinositol (GPI) of brain from both the sunflower seed oil and fish oil group, although the fatty acid was existed in the total IGP fraction $(2.2$ and $5.6 \mathrm{~mol} \%$, respectively) (Fig.2). Whereas markedly higher levels of the polyunsaturated fatty acids as arachidonic acid and docosahexaenic acid were exhibited in diacyl-GPI subclass of the sunflower seed oil and fish oil animals (Fig. 2). The docosahexaenoic acid provided the greatest contribution to the higher levels of n-3 fatty acids in the diacyl-GPI from the two dietary groups (2.1 and $2.8 \mathrm{~mol} \%$, respectively). The docosapentaenoic acid provided for a lesser portion (and docosahexaenoic acid for a greater portion) of the n-3 fatty acids in both the sunflower seed oil and fish oil groups in the diacyl types of IGP. The docosapentaenoic acid and docosahexaenic acid showed a significant elevation in diacyl
Table 4. Acyl chain composition of inositol glycerophospholipid in brain of rats fed either a sunflower seed oil (SO) or fish oil (FO) $\operatorname{diets}^{\mathrm{a}}$

\begin{tabular}{|c|c|c|}
\hline \multirow{2}{*}{ Acyl chain } & \multicolumn{2}{|c|}{ Composition (mol \%) } \\
\hline & $\mathrm{SO}$ & $\mathrm{FO}$ \\
\hline $14: 0$ & $3.8 \pm 1.0$ & $2.7 \pm 0.7$ \\
\hline $14: 1$ & $0.1 \pm 0.1$ & $0.1 \pm 0.1$ \\
\hline $16: 0$ & $9.0 \pm 0.7$ & $9.0 \pm 0.6$ \\
\hline $16: 1$ (iso) $^{b}$ & $\operatorname{tr}$ & $\operatorname{tr}$ \\
\hline $18: 0$ & $49.1 \pm 6.9$ & $48.2 \pm 5.1$ \\
\hline $18: 1$ (iso) $^{\mathrm{b}}$ & $7.2 \pm 0.9$ & $6.4 \pm 0.5$ \\
\hline $18: 2 n-6$ & $6.5 \pm 1.0$ & $4.1 \pm 0.4$ \\
\hline $18: 3 n-3$ & $\operatorname{tr}$ & $0.7 \pm 0.7$ \\
\hline $20: 0$ & $\operatorname{tr}$ & $\operatorname{tr}$ \\
\hline $20: 1$ & $\operatorname{tr}$ & $0.1 \pm 0.1$ \\
\hline $20: 2 n-6$ & $\operatorname{tr}$ & $\operatorname{tr}$ \\
\hline $20: 3 n-6$ & $\operatorname{tr}$ & $\operatorname{tr}$ \\
\hline $20: 4 n-6$ & $21.1 \pm 5.6$ & $20.7 \pm 2.6$ \\
\hline $20: 3 n-3$ & $\operatorname{tr}$ & $\operatorname{tr}$ \\
\hline $20: 5 n-3$ & $\operatorname{tr}$ & $1.0 \pm 0.1^{\mathrm{d}}$ \\
\hline $22: 0$ & $\operatorname{tr}$ & $\operatorname{tr}$ \\
\hline $22: 1$ & $\operatorname{tr}$ & $0.2 \pm 0.1$ \\
\hline $22: 3 n-6$ & $\operatorname{tr}$ & $\operatorname{tr}$ \\
\hline $22: 4 n-6$ & $\operatorname{tr}$ & $\operatorname{tr}$ \\
\hline $22: 5 n-6$ & $\operatorname{tr}$ & $\operatorname{tr}$ \\
\hline $22: 5 n-3$ & $\operatorname{tr}$ & $0.3 \pm 0.1$ \\
\hline $22: 6 n-3$ & $2.2 \pm 0.8$ & $5.6 \pm 0.7^{\mathrm{c}}$ \\
\hline Unidentified. & $1.1 \pm 0.1$ & $1.0 \pm 0.1$ \\
\hline
\end{tabular}

${ }^{a}$ Values represent means \pm S.E. for seven animals/group.

biso means isomers.

'Indicates significant difference from the $\mathrm{CO}$ group at the level $p<0.05$.

${ }^{\mathrm{d}}$ Indicates significant difference from the $\mathrm{CO}$ group at the level $p<0.01$.

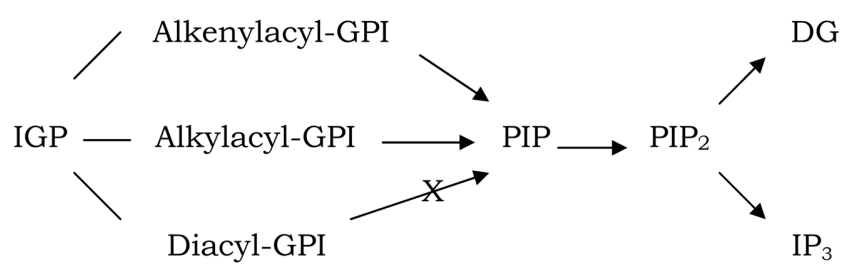

Fig. 1. The possible signaling transduction pathway of etherlinked inositol glycerophospholipids to produce polyphosphoinositides in rat brain.

subclass of IGP from the brain of the sunflower seed oil and fish oil animals; a greater accumulation was found in the sunflower seed oil (1.4 and $2.1 \mathrm{~mol} \%$ relative to 1.4 and 2.8 $\mathrm{mol} \%$ of the fish oil group.

Docosapentaenoic acid and docosahexaenoic acid were not found in the acyl-chain composition of phosphatidylinositol4-phosphate (PIP) and phosphatidylinositol-4, 5-bisphosphate 


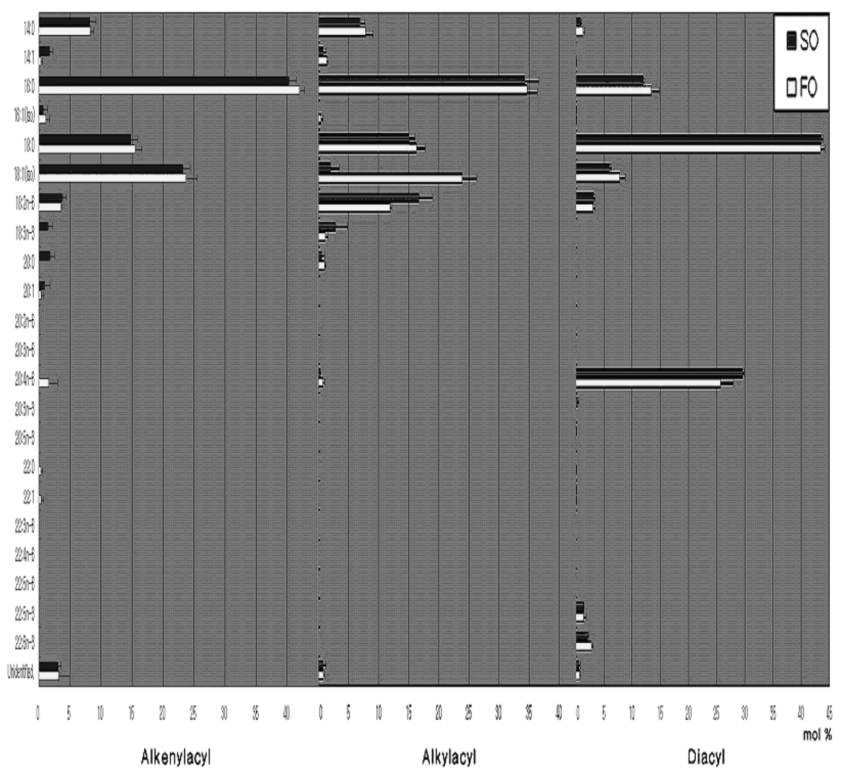

Fig. 2. Acyl chain composition of alkenylacyl, alkylacyl and diacyl subclasses of inositol glycerophospholipids in brain of rats fed either a sunflower seed oil (SO) or fish oil (FO) diets.

Values represent means \pm S.E. for seven animals/group. Iso means isomers.

*Indicates significant difference from the $\mathrm{CO}$ group at the level $p<0.05$.

**Indicates significant difference from the $\mathrm{CO}$ group at the level $p<0.01$.

$\left(\mathrm{PIP}_{2}\right)$ in brain of rats fed either a sunflower seed oil or fish oil diets (Fig. 3) as shown in Fig. 2 for alkenylacyl- and alkylacyl-GPI, respectively.

\section{Discussion}

No docosapentaenoic acid and docosahexaenoic acid were found in alkenylacyl- and alkylacyl-GPI of brain from both the sunflower seed oil and fish oil group, though the fatty acid was existed in the total IGP and diacyl-GPI fraction from the two dietary groups (Table 4, Fig. 2). The docosahexaenoic acid, which was found in considerably high levels (both in the sunflower seed oil and fish oil animals) in the diacyl-GPI as compared to the alkenylacyl- and alkylacyl-GPI. This finding is in disagreement with the reported enrichment of the n-3 fatty acids in the alkylacyl and alkenylacyl classes of individual phospholipid in other tissues from animals fed fish oil-based diets (Yeo, Y.K et al., 1988; Yeo et al., 1989; Yeo et al., 1989; Yeo and Holub, 1990; Park et al., 1995; Yeo et al., 1999; Yeo et al., 2000) and suggests that one effect of dietary oil feeding was a different metabolism via bio-availability of docosapentaenoic acid and docosahexaenoic acid in brain IGP.

Interestingly, considerable enrichment of docosapentaenoic acid and docosahexanoic acid was found in the diacyl-IGP of

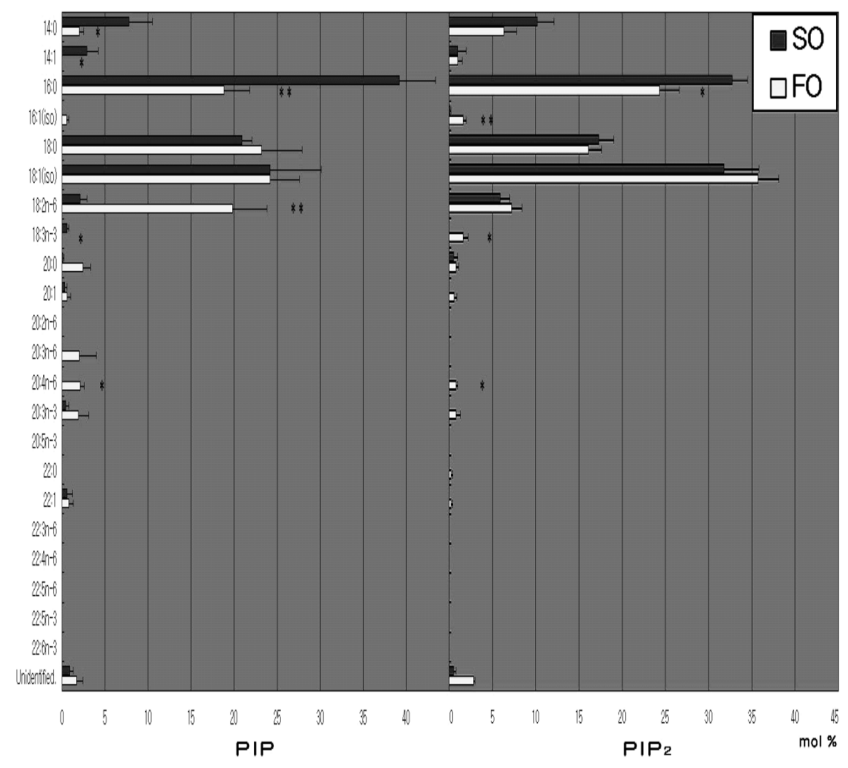

Fig. 3. Acyl chain composition of phosphatidylinositol-4-phosphate (PIP), phosphatidylinositol-4,5-bisphosphate $\left(\mathrm{PIP}_{2}\right)$ in brain of rats fed either a sunflower seed oil (SO) or fish oil (FO) diets.

Values represent means \pm S.E. for seven animals/group. Iso means isomers.

*Indicates significant difference from the $\mathrm{CO}$ group at the level $p<0.05$.

**Indicates significant difference from the $\mathrm{CO}$ group at the level $p<0.01$.

the sunflower seed oil and fish oil groups. A possible discrimination against docosapentaenoic acid and docosahexaenoic acid release from the diacyl-GPI of membrane IGP via the phospholipase $\mathrm{A}_{2}$ enzyme might be pronounced in rat brain.

Results from this study indicate that dietary ingestion of the sunflower seed oil and fish oil concentrate to rats was associated with only the diacyl-GPI species of IGP in the brain relative to ether-linked IGP fractions in this tissue. Brains from the two oil-fed rats showed no docosapentaenoic acid and docosahexaenoic acid in alkenylacyl- and alkylacylGPI although the oil enriched with docosahexaenoic acid was fed to the animal. The docosapentaenoic acid in the etherlinked IGP fractions seems to be retroconverted from docosahexaenoic acid which was not contained enough in the either dietary sunflower seed oil or fish oil.

This is also apparent from the $22: 5 n-3 / 22: 6 n-3$ molar ratios of 0.67 and 0.50 in the diacyl-GPI, respectively, in the sunflower seed oil and fish oil animals. In contrast, the docosapentaenoic acid and docosahexaenoic acid in the etherlinked IGP were not found at all in the two dietary groups.

The inositol phospholipids comprise less than $10 \%$ of the total phospholipids in mammalian cell membranes. The IGP, as a minor phospholipid in cell membranes found to be most important in signal transduction, were two phosphorylated derivatives of IGP, PIP and $\mathrm{PIP}_{2}$. No polyunsaturated fatty acids (docosapentaenoic acid and docosahexaenoic acid) were 
found in the two derivatives of IGP (PIP and $\mathrm{PIP}_{2}$ in Fig. 3) as in the ether-linked IGP (alkenylacyl- and alkylacyl-GPI).

A role for inositol phospholipids in signal transduction is considerably well understood (Darnell et al., 1991; Chilvers et al., 1991; Ohnuma and Mabuchi, 1993; Englund, 1993; Munnik et al., 1994). The chain of events linking extracellular signals to intracellular responses via $\mathrm{PIP}_{2}$ breakdown begins with binding of the signaling molecules to its receptor in the plasma membrane. Although the details of the activation processes are not as well known as they are in the cyclic AMP pathway, there is increasing evidence that the same type of multistep mechanism operates in the plasma membranes (Yamauchi et al., 1993; Johnso et al., 1993; Sen and Chander, 1994; Palmer et al., 1994; Rameh et al., 1997). The polyphosphoinositides (PIP and $\mathrm{PIP}_{2}$ ) are produced by the phosphorylation of IGP. Although all three inositol phospholipids may be hydrolyzed in the signaling responses, it is the breakdown of $\mathrm{PIP}_{2}$ that is most critical, even though it is the least abundant, constituting less than $10 \%$ of the total inositol lipids and less than $1 \%$ of the total phospholipids (Granoff, 1987; Chilvers et al., 1991; Darnell et al., 1991).

Of particular interest in the present work are the result showing no docosapentaenoic acid and docosahexaenoic acid content in the ether-linked IGP and polyphosphoinositides (PIP and $\mathrm{PIP}_{2}$ ) which are produced by the phosphorylation of IGP from the two dietary oil group. These results are in general agreement with Alsted and Hoy (1992) who found little docosahexaenoic acid in PIP and $\mathrm{PIP}_{2}$ fractions compared to total inositol glycerophospholipid in brain of rat which fed with three different oils including fish oil enriched with docosahexaenoic acid. Their results suggest that PIP and $\mathrm{PIP}_{2}$ are possibly from only ether-linked IGP because the diacyl-GPI is a major subclass among 3-subclasses (alkenylacyl, alkylacyl and diacyl) of IGP. In view of the result so far achieved, the study strongly suggests that the polyphosphoinositides might be phosphorylated from the ether-linked IGP (alkenylacyland alkylacyl species) instead of diacyl subclass for the chain of events linking extracellular signals to intracellular responses in the plasma membrane of rat brain (Fig. 1).

The specific biochemical reactions involved in the biosynthesis and turnover of the alkenylacyl and alkylacyl species of IGP, including those specifically reported in the brain that are phosphorylated to generate the polyphosphoinositides (PIP and PIP 2 ), remain to be studied. As well, more study is required to determine the effects of dietary fatty acids on the biosyntheses of the phosphoinositides as well as the possible role of these biochemical changes on brain.

Acknowledgments This study was supported by a research grant from the Kyungpook National University and Ministry of Agriculture, Korea. We thank Dr. Diana-Jean Philbrick for reviewing the manuscript.

\section{References}

Alsted, A.-L. and Hoy, C.-E. (1992) Fatty acid profiles of brain phospholipid subclasses of rats fed n-3 polyunsaturated fatty acids of marine or vegetable origin. A two generation study. Biochim. Biophys. Acta 1125, 237-244.

Bernett, J. T., Meredith, N. K., AKins, J. R. and Hannon, W. H. (1985) Determination of srum phospholipid metabolic profiles by high performance liquid chromatography. J. Liquid Chromatogr. 8, 1573-1591.

Berridge, M. J. (1993) Inositol triphosphate and calcium signaling. Nature 361, 315-326.

Chaffoy de Courcelles, D., Roevens, P. and Van Belle, H. (1984) 12-O-Tetradecanoylphorbol 13-acetate stimulates inositol lipid phosphorylation in intact human platelets. FEBS Lett. 173, 389390.

Chilvers, E. R., Batty, I. H., Challiss, R. A. J. and Barnes, P. J. (1991) Determination of mass changes in phosphatidylinositol 4,5-isphosphate and evidence for agonist-stimulated metabolism of inositol 1,4,5-triphosphate in airway smooth muscle. Biochem. J. 275, 373-379.

Curstedt, T. (1983) Chromatographic analysis, isolation and characterization of ether lipids; in Ether Lipids, Mangold, H. K. and Paltauf, F. (eds.), pp. 1-15, Academic Press, New York, USA.

Darnell, J. C., Osterman, D. G. and Saltiel, A. R. (1991) Synthesis of phosphatidylinositol in rat liver microsomes in accompanied by the rapid formation of lysophosphatidylinositol. Biochim. Biophys. Acta 1084, 269-278.

Englund, P. T. (1993) The structure and biosynthesis of glicosyl phosphatidylinositol protein anchors. Annu. Rev. Biochem. 62, 121-138.

Granoff, B. W. (1987) Inositol lipid turnover in the central nervous system; in Inositol Lipids in Cellular Signaling, Michell, R. H. and Putney, J. W. (eds.), pp. 163-165, Cold Spring Harbor, New York, USA.

Holub, B. J. and Skeaff, C. M. (1987) Nutritional regulation of cellular phosphatidylinositol. Methods Enzymol. 141, 234-244.

Johnso, S. C., Dahl, J., Shih, T. L., Schedler, D. J. A., Anderson, L., Benjamin, T. L. and Baker, D. C. (1993) Synthesis and evalution of 3-modified 1D-myo-inositols as inhibitors and substrates of phosphatidylinositol synthase and inhibitors of myo-inositol uptake by cells. J. Med. Chem. 36, 3628-3635.

Low, M. G. and Saltiel, A. R. (1988) Structural and functional roles of glycosyl-phosphatidylinositol in membranes. Science 239, 268-275.

Medh, J. D. and Weigel, P. H. (1989) Separation of phosphatidylinositols and other phospholipids by two-step onedimensional thin-layer chromatography. J. Lipid Res. 30, 761764.

Munnik, T., Irvine, R. F. and Musgrave, A. (1994) Rapid turnover of phosphatidylinositol 3-phosphate in the green alga chlamydomonas eugametos: signs of a phosphatidylinositide 3kinase signalling pathway in lower plants? Biochem. J. 298, 269-273.

Ohnuma, M. and Mabuchi, I. (1993) 45K Actin filament-severing protein from sea urchin eggs: Interaction with phosphatidylinositol- 
4,5-bisphosphate. J. Biochem. 114, 718-722.

Palmer, F. B., Theolis R. Jr., Cook, H. W. and Byers, D. M. (1994) Purification of two immunologically related phosphatidylinositol-4,5-bisphosphate phosphatases from bovine brain cytosol. J. Biol. Chem. 269, 3403-3410.

Park, J. H., Park, E. J., Kim, K. S. and Yeo, Y. K. (1995) Changes in ether-linked phospholipids in rat kidney by dietary alpha-linolenic acid in vivo. Lipids 30, 541-546.

Rahmant, S. M., Huda, M. N., Uddin, M. N. and Akhteruzzaman, S. (2002) Short-term administration of conjugated linoleic acid reduces liver triglyceride concentration and phosphatidate phosphohydrolase activity in OLETF rats. J. Biochem. Mol. Biol. 35, 494-497.

Rameh, L. E., Tolias, K. T., Duckworth, B. and Cantley, L. C. (1997) A new pathway for synthesis of phosphatidylinositol4,5-bisphosphate. Nature 390, 192-196.

Sen, N. and Chander, A. (1994) Alkalosis- and ATP-induced increases in the diacylglycerol pool in alveolar type II cells are derived from phosphatidylcholine and phosphatidylinositol. Biochem. J. 298, 681-687.

Yamauchi, K., Holt, K. and Pessin, J. E. (1993) Phosphatidylinositol 3-kinase functions upstream of ras and raf in mediating insulin stimulation of c-fos transcription. J. Biol. Chem. 268, 14597-14600.

Yasutomi, N. (1992) Intracellular sinaling by hydrolysis of phospholipids and activation of protein C. Science 258, 607614.
Yeo, Y. K., Philbrick, D. J. and Holub, B. J. (1988) Altered acyl chain compositions of alkylacyl, alkenylacyl, and diacyl subclasses of choline and ethanolamine glycerophospholipids in rat heart by dietary fish oil. Biochim. Biophys. Acta 1001, 2530.

Yeo, Y. K., Philbrick, D. J. and Holub, B. J. (1989) Effects of dietary $\mathrm{n}-3$ fatty acids on mass changes and $[3 \mathrm{H}]$ glycerol incorporation in various glycerolipid classes in rat kidney in vivo. Biochim. Biophys. Acta 1006, 9-14.

Yeo, Y. K., Philbrick, D. J. and Holub, B. J. (1989) The effect of dietary n-3 fatty acid on the incorporation of radioactivity into ether-linked renal phospholipids in rats following [114C]hexadecanol injection; in Health Effects of Fish and Fish oil, Chandra, R. K. (ed.), pp. 525-536, ARTS Biochemical Publishers, St. John's, Canada.

Yeo, Y. K. and Holub, B. J. (1990) Influence of dietary fish oil on the relative syntheses of triacylglycerol and phospholipids in rat liver in vivo. Lipids 25, 811-814.

Yeo, Y. K., Lim, A. Y., Lee, J. Y., Kim, H. J., Tibor, F. and Kim, D. K. (1999) Eicosapentaenoic and docosahexaenoic acids reduce arachidonic acid release by rat kidney microsomes. $J$. Biochem. Mol. Biol. 32, 33-38.

Yeo, Y. K., Kim, J. S., Lee, J. R., Lee, J. Y., Chung, S. W., Kim, H. J., Horrocks, L. A. and Park, Y. S. (2000) Plasma phospholipids, including plasmalogens after consumption of diet enriched in long-chain n-3 fatty acids. J. Biochem. Mol. Biol. 33, 499-505. 